\title{
Spin control using chiral templated nickel $\odot$
}

Cite as: Appl. Phys. Lett. 118, 224001 (2021); https://doi.org/10.1063/5.0049113

Submitted: 01 March 2021 . Accepted: 26 April 2021 . Published Online: 01 June 2021

(D) Suryakant Mishra, (D) Luca Pasquali, and (D) Claudio Fontanesi

\section{COLLECTIONS}

F This paper was selected as Featured
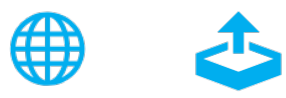

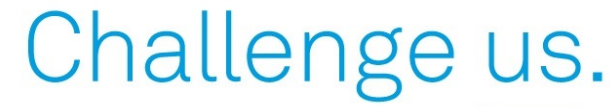

What are your needs for periodic signal detection?

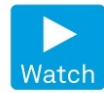

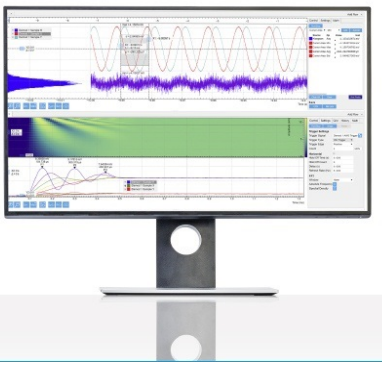

Zurich

Instruments 


\title{
Spin control using chiral templated nickel $\odot$
}

\author{
Cite as: Appl. Phys. Lett. 118, 224001 (2021); doi: 10.1063/5.0049113 \\ Submitted: 1 March 2021 - Accepted: 26 April 2021 • \\ Published Online: 1 June 2021 \\ Suryakant Mishra, $^{1,2,3, a)}$ (D) Luca Pasquali,, ${ }^{4,5}$ (D) and Claudio Fontanesi ${ }^{4, a)}$ (iD \\ AFFILIATIONS \\ ${ }^{7}$ Pritzker School of Molecular Engineering at University of Chicago, 5640 S Ellis Ave, Chicago, Illinois 60637, USA \\ ${ }^{2}$ Center for Nanoscale Materials, Argonne National Laboratory, Lemont, Illinois 60439, USA \\ ${ }^{3}$ Department of Chemical and Biological Physics, Weizmann Institute of Science, Rehovot 76100, Israel \\ ${ }^{4}$ Dipartimento di Ingegneria Enzo Ferrari, Via Vivarelli 10, Modena 41125, Italy \\ ${ }^{5}$ OM-CNR, Strada Statale 14, Km. 163.5 in AREA Science Park, Basovizza, Trieste 34149, Italy
}

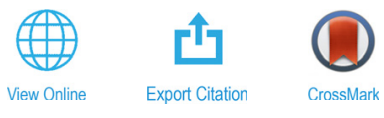

${ }^{a)}$ Authors to whom correspondence should be addressed: mishras@uchicago.edu and claudio.fontanesi@unimore.it

\begin{abstract}
This Letter reports an original spin valve device that is based on a chiral templated nickel material. Chirality in Ni is induced by exploiting co-electrodeposition of an organic chiral template. In this specific case, the chiral templating is enantiopure tartaric acid (TA). Facile electrodeposition (co-deposition) in ambient conditions produces a nickel chiral-templated material. Z-shaped magnetoresistance curves, switching sign as a function of TA handedness, prove the peculiar ferromagnetic character induced by the presence of a chiral compound. Synchrotron measurements using circular polarized light, x-ray natural circular dichroism, confirm the chirality of the Ni in the TA/Ni composite. Density functional theory calculation proves the existence of a strong electronic delocalization involving the tartaric acid and Ni. The significant finding of this Letter is that chiral templated Ni paves the way for future spin valve, which will be able to control the spin without an external magnetic field (as indeed foreseen within the chiral induced spin selectivity-effect framework).
\end{abstract}

Published under an exclusive license by AIP Publishing. https://doi.org/10.1063/5.0049113

Use of the "spin," in information storage devices rather than charge, paves the way to a dramatic reduction in power consumption. $^{1-3}$ Spintronic is a game-changing strategy for minimizing heat dissipation, a crucial issue for electronic devices. Indeed, most of the recent developments in spintronics rely on the implementation of the giant magnetoresistance (GMR) effect in spin-based logic gates. ${ }^{4}$ Remarkable progress has been seen in the past couple of years, concerning interlayer exchange coupling, ${ }^{5}$ spin-transfer torque (STT), ${ }^{6}$ and large tunneling magnetoresistance (TMR). ${ }^{7}$ Spin polarization can also be achieved even without magnetic moment just by utilizing spin orbital coupling (SOC) or Rashba by Spin Hall effects (SHE). These techniques recently emerged as a promising source of torques to manipulate neighboring magnetic nanostructures efficiently and are suitable for many related applications (such as nonvolatile memory, logic-gate, and supercomputer quantum bit or Qbit). ${ }^{8}$

Within this field it has to be noted, the experimental result suggests a tight connection between the electron spin and the most intriguing, and yet a challenging area, of "asymmetry in nature," i.e., chirality, as proposed by Professor Ron Naaman: chiral induced spin selectivity (CISS) effect.' Due to the CISS-effect, transmission of one spin is preferred by one chirality, and vice versa for the opposite spin. In this area of scientific research, there are significant number of studies focused on organic polymers, ${ }^{10-13}$ supramolecular structure, ${ }^{14}$ and biological systems such as DNA, ${ }^{15,16}$ proteins, ${ }^{17}$ and oligopeptides. ${ }^{16,18,19}$ Electron transport in these systems, which feature self-assembled monolayers (SAMs) of chiral organic compounds, is found to be spin selective.

Here, we demonstrate that, relying on CISS, an inorganic based chiral system, where chirality is induced by tartaric acid (TA), can be used to fabricate a spin selective device. ${ }^{20}$ To carry out magnetoresistance (MR) measurements, a standard 4-probe method is used: the change in resistance is measured as a function of the magnetic field $(B)$. MR curves as a function $B$ show an opposite pattern, upon switching the TA handedness. This MR device, where one ferromagnetic layer is replaced by a chiral layer called as "modify" MR device or spin valve, is able to measure the amount of spin injection/scattering in/ from a chiral ferromagnetic layer. This device would help to enable future research on a spin valve without an external magnetic field. In contrast to the previous work based on the use of well-ordered chiral molecule monolayers, in the present paper chiral $\mathrm{Ni}$ is obtained by a simple, bulk, co-electrodeposition. The electrodeposited Ni is obtained as a mesoscopic (about $0.3 \mu \mathrm{m}$ thickness) uniform compact and pinhole free layer. Remarkably, MR facts and figures are found comparable with SAM based spin-filtering systems, but our hybrid TA/Ni device remains stable for a longer time and it is definitely simpler to 
produce. In general, controlling chirality by electrochemical codeposition (the chiral compound is simply added in a bulk solution) proves to be a more simple and efficient technique: one can easily control the system handedness, i.e., spin-filtering, by selecting a suitable organic chiral template. ${ }^{11,21-24}$

In particular, chirality in $\mathrm{Ni}$ is induced by electrochemical co-deposition from a Ni/TA solution, just adding enantiopure TA to a Watts bath (more detail is available in the supplementary material). ${ }^{25}$ Figure 1(a) shows schematically the single drop electrochemical setup for the deposition of chiral nickel. Figure 1(b) shows a typical cyclic voltammetry $(\mathrm{CV})$ curve obtained from a nickel-Watts/TA bath. The $\mathrm{CV}$ curve features a preeminent oxidation peak at about $0.5 \mathrm{~V}$ in the forward (positive direction) scan, rounded by the relevant reduction peak at $0.26 \mathrm{~V}$ in the backward scan. These two peaks correspond to the reversible $\mathrm{NiO}(\mathrm{OH})+e^{-}+\mathrm{H}_{2} \mathrm{O} \leftrightharpoons \mathrm{Ni}(\mathrm{OH})_{2}+\mathrm{OH}^{-}$redox process. $^{21,24}$ Figure 1 (c) shows a typical chronoamperometric response curve relevant to the electrodeposition carried out at a $-1.4 \mathrm{~V}$ constant potential for $150 \mathrm{~s}$, the same solution composition as in Fig. 1(b).

Chiral templated nickel is used in the MR spintronics device, as depicted in Fig. 2(a), where the chiral Ni is electrodeposited on the

(A)
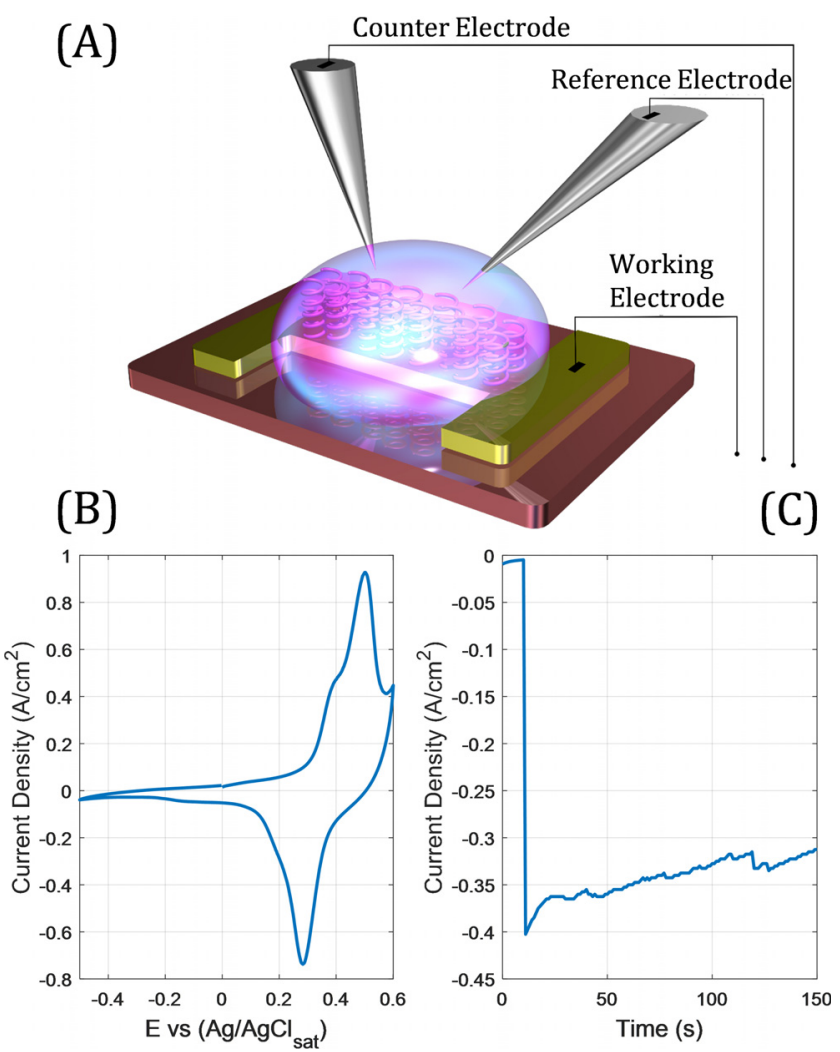

FIG. 1. (a) Schematic representation of the experimental setup used for electrodeposition of templated chiral Ni: the working electrode (at the bottom of the drop) is a $0.5 \mathrm{~mm} \times 2 \mu \mathrm{m}$ gold surface. (b) Cyclic voltammetry curve (CV) of a Watts bath (details in the supplementary material) with added enantiopure $0.1 \mathrm{M}$ TA on a gold working electrode, $50 \mathrm{mV} / \mathrm{s}$ the scan rate; a Pt wire is the counter electrode (CE), $\mathrm{Ag} / \mathrm{AgCl} / \mathrm{KCl}_{\text {sat }}$, is the reference (RE). (C) Chronoamperometry (CA) curve obtained during potentiostatic, $E=-1.4 \mathrm{~V}, \mathrm{Ni} / \mathrm{TA}$ co-deposition. bottom gold electrode. As a whole, the MR device is finished implementing a standard 4-probe measurement configuration by using a suitable current source and a voltage meter; an out-of-plane magnetic field [red solid arrow marked with B in Fig. 2(a), orthogonal to the MR device surface] is applied to switch the magnetization direction and intensity (device fabrication details, and relevant geometrical structure, are reported in the supplementary material). Inset of Fig. 2(a) presents the CISS-effect based spin-polarization in chiral templated right and left-handed Ni. The change in resistance is measured with respect to the applied magnetic field and then plotted in terms of MR percentage

$$
\operatorname{MR}(\%)=\frac{R_{M}-R_{0}}{R_{0}} \times 100 .
$$

Figure 2(b) shows MR response of achiral Ni as a cross check, and MR values decrease in a symmetrical way as a function of both the directions of $B$ : symmetrical spin alignment from random to parallel, without any asymmetry as a function of $B$ direction (no spin-discrimination). Remarkably, Figs. 2(c) and 2(d), L-Ta/Ni and D-Ta/Ni, respectively, are characterized by Z-shaped MR curves, featuring an opposite polarity. [Figures 2(c) and 2(d) are mirroring graphs as a function of the magnetic field direction, i.e., sign.] This gives clear indication of intrinsic spin filtering capability (the sign of the MR signal is a function of the magnetic field orientation) of our chiral templated-nickel based MR device. In the case of L-TA, the resistance is higher when magnetization is north polarized and resistance gets lower when the magnetization direction is switched. The D-TA/Ni based device shows an opposite behavior. This is one of the important aspects, a quite peculiar result, of this work: control of Ni handedness, controls spin transmission (or for a given $\mathrm{Ni}$ handedness, the magnetic field direction controls the resistance).

NEXAFS XNCD measurements, recorded at the Ni $L_{3}$ edge, Fig. 3, feature an evident dichroic behavior. This evidence gives due reason to the effective ability of TA in inducing chirality in the nickel. It is important to appreciate the fact that, by normalizing the baseline in Fig. 3, we observed a clear difference in the intensities in D-TA/Ni and L-TA/Ni samples.

Figure 4 summarizes theoretical results that are related to the XNCD response. Figures 4(a) and 4(b) show the full-optimized guessmodel molecular complexes formed between a Ni atom and two L-TA molecules in a "squared" and "planar" like geometrical configuration, $1 \mathrm{~mol}$ and $2 \mathrm{~mol}$, respectively. $1 \mathrm{~mol}$ is found about $20 \mathrm{kcal} \mathrm{mol}^{-1}$ lower in energy than the $2 \mathrm{~mol}$ species. $1 \mathrm{~mol}$ and $2 \mathrm{~mol}$ species are obtained from full optimization of a square and planar initial Ni/TA geometries inspired by the results of Hoek and Sachtler. ${ }^{26}$ Indeed, both the squared and planar geometries here presented correspond to true minimum on the potential energy surface. As proved by the hessian calculation following a full geometry optimization: all the calculated frequencies are real and positive. Figures 4(c) and (d) show the Kohn-Sham LUMO orbitals for $1 \mathrm{~mol}$ and $2 \mathrm{~mol}$, respectively. It has to be noted that the NEXAFS signal probes virtual orbitals, and at the threshold energy for electron excitation, due to the probing photon energy, a core electron is excited from a core atomic orbital to the lowest in energy available orbital, i.e., the LUMO. Figures 4(e) and 4(f) set out UV-Vis and CD for $1 \mathrm{~mol}$ and $2 \mathrm{~mol}$, respectively. Indeed, a classical LUMO $\leftarrow$ HOMO transition underlies the $600 \mathrm{~nm}$ peak (also optical active), which is evident in both Figs. 4(e) and 4(f). 


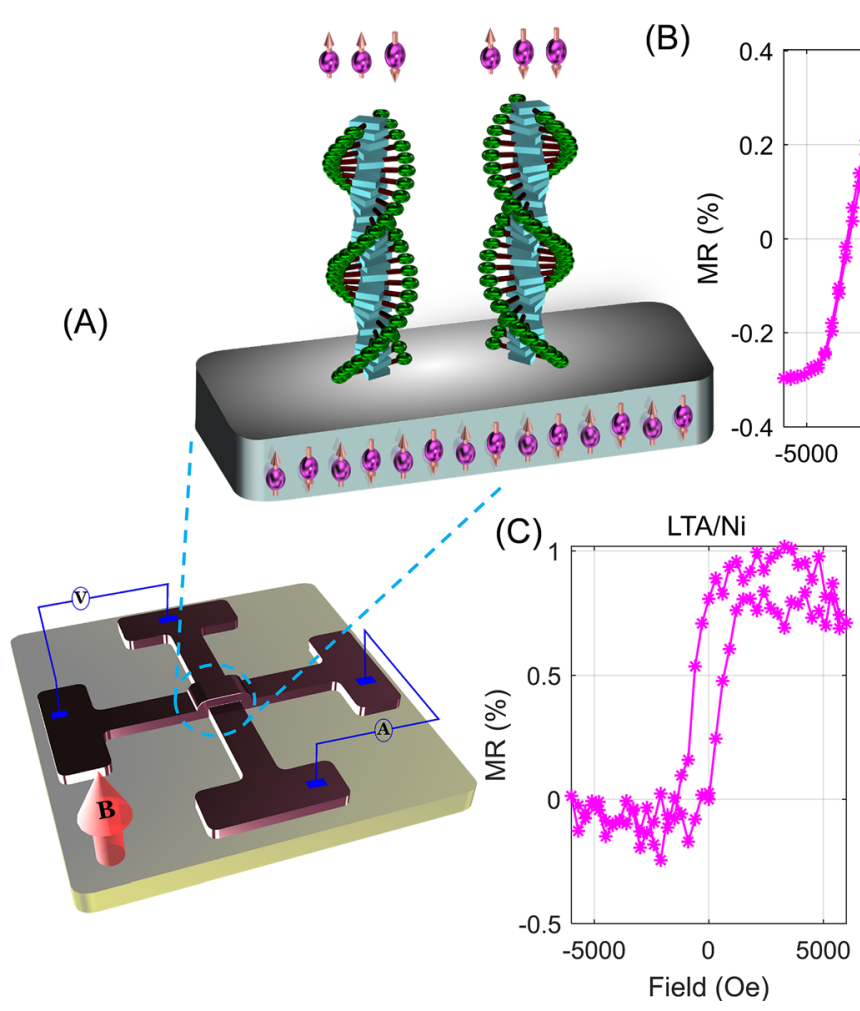

Figures 4(e) and 4(f) set out theoretical spectra relevant to the $1 \mathrm{~mol}$ and $2 \mathrm{~mol}$ complexes, respectively. Remarkably, both the $1 \mathrm{~mol}$ and 2 mol LUMOs are evenly delocalized on both the nickel and TA moieties, compare Figs. 4(c) and 4(d), see the supplementary material for additional details regarding the numerical and experimental methods.

We report on the peculiar MR results of the original material, $\mathrm{c}-\mathrm{Ni}$, which demonstrate as an appealing hybrid composite for the realization of a magnetic field less spin valve for future spintronics application. Additionally, electrochemical preparation is rather simple and cheap, yielding a material rather robust under usual laboratory ambient conditions. Chirality in Ni using an organic chiral catalyst is

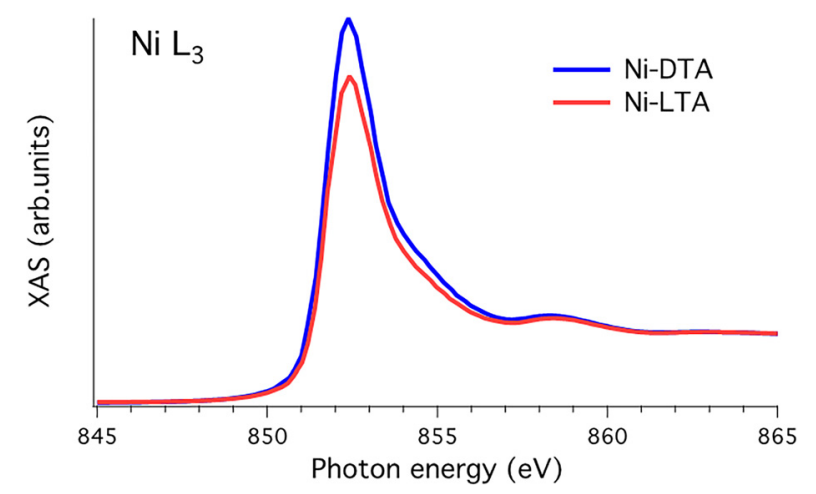

FIG. 3. X-ray natural circular dichroism (XNCD) spectra at the $\mathrm{Ni} L_{3}$ edge. Blue curve: D-TA/Ni. Red curve: L-TA/Ni.
FIG. 2. (a) Schematic representation of the magnetoresistance (MR) device, experimental setup: a standard 4-probe configuration is implemented. (b) MR plot with a magnetic field measured on achiral $\mathrm{Ni}$ as a cross-check experiment. (c) and (d) on chiral Ni, L-TA and D-TA, respectively.

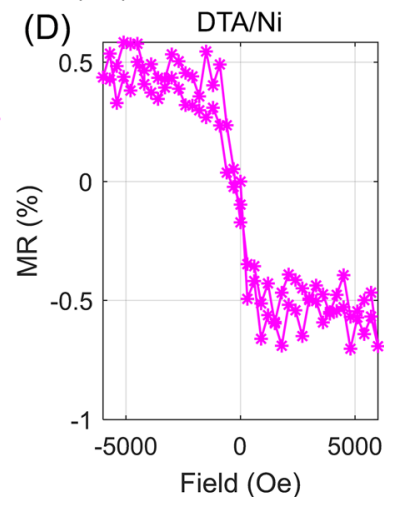

(A)

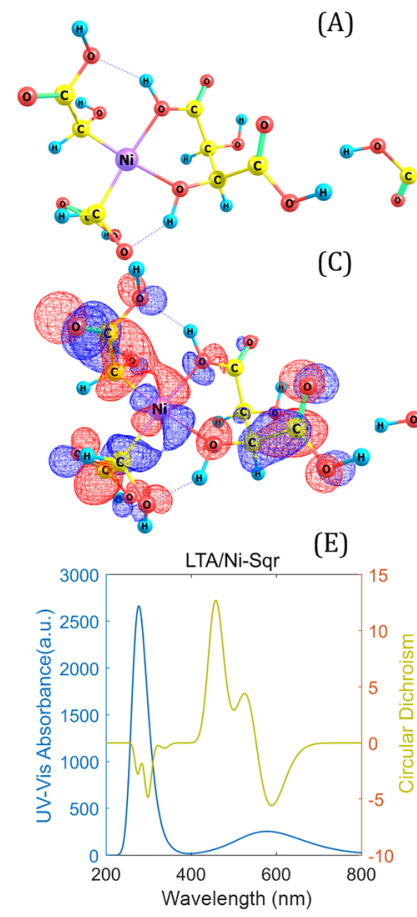

(B)

FIG. 4. (a) and (b) Schematic presentation of the molecular models for $1 \mathrm{~mol}$ and 2 mol Ni complex with two TA molecules. (c) and (d) Relevant LUMO distribution. (e) and (f) Calculated, B3LYP/6-31G(d), electronic absorption, and CD spectra. 
proven to be an efficient tool, where one can easily control the right and left handedness. Synchrotron measurement confirms correlation of helicity with electron emission through circular polarized light.

See the supplementary material that includes details of experimental setup, steps involve in device fabrication, and information about theoretical calculation.

S.M. and C.F. are deeply thankful to Professor Ron Naaman for the useful discussion on CISS-effect. S.M. appreciates the help of Dr. Gregory Leitus (Weizmann Institute of Science) for discussion on MR measurement. C.F. gratefully acknowledges "Joseph Meyerhoff Visiting Professorship" at the Weizmann Institute of Science.

\section{DATA AVAILABILITY}

The data that support the findings of this study are available from the corresponding author upon reasonable request.

\section{REFERENCES}

${ }^{1}$ I. Žutić, J. Fabian, and S. Das Sarma, Rev. Mod. Phys. 76, 323 (2004).

${ }^{2}$ A. Fert, Rev. Mod. Phys. 80, 1517 (2008).

${ }^{3}$ C. Chappert, A. Fert, and F. N. Van Dau, in Nanoscience and Technology (CoPublished with Macmillan Publishers Ltd, UK, 2009), pp. 147-157.

${ }^{4}$ M. N. Baibich, J. M. Broto, A. Fert, F. N. Van Dau, F. Petroff, P. Etienne, G. Creuzet, A. Friederich, and J. Chazelas, Phys. Rev. Lett. 61, 2472 (1988).

${ }^{5}$ A. Chernyshov, M. Overby, X. Liu, J. K. Furdyna, Y. Lyanda-Geller, and L. P. Rokhinson, Nat. Phys. 5, 656 (2009).

${ }^{6}$ I. M. Miron, K. Garello, G. Gaudin, P.-J. Zermatten, M. V. Costache, S. Auffret, S. Bandiera, B. Rodmacq, A. Schuhl, and P. Gambardella, Nature 476, 189 (2011).

${ }^{7}$ S. S. P. Parkin, C. Kaiser, A. Panchula, P. M. Rice, B. Hughes, M. Samant, and S.-H. Yang, Nat. Mater. 3, 862 (2004).

${ }^{8}$ C. Nayak, S. H. Simon, A. Stern, M. Freedman, and S. Das Sarma, Rev. Mod. Phys. 80, 1083 (2008).
${ }^{9}$ K. Ray, S. P. Ananthavel, D. H. Waldeck, and R. Naaman, Science 283, 814 (1999).

${ }^{10}$ S. Mishra, A. K. Mondal, E. Z. B. Smolinsky, R. Naaman, K. Maeda, T. Nishimura, T. Taniguchi, T. Yoshida, K. Takayama, and E. Yashima, Angew. Chem. Int. Ed. 59(34), 14671-14676 (2020).

${ }^{11}$ S. Mishra, A. Kumar, M. Venkatesan, L. Pigani, L. Pasquali, and C. Fontanesi, Small Methods 4, 2000617 (2020).

${ }^{12}$ P. Morvillo, R. Diana, C. Fontanesi, R. Ricciardi, M. Lanzi, A. Mucci, F. Tassinari, L. Schenetti, C. Minarini, and F. Parenti, Polym. Chem. 5, 2391 (2014).

${ }^{13}$ F. Allegretti, V. De Renzi, R. Biagi, U. del Pennino, G. Contini, V. D. Castro, C. Mariani, M. G. Betti, and C. Fontanesi, Surf. Sci. 539, 63 (2003).

${ }^{14}$ W. Mtangi, F. Tassinari, K. Vankayala, A. Vargas Jentzsch, B. Adelizzi, A. R. A. Palmans, C. Fontanesi, E. W. Meijer, and R. Naaman, J. Am. Chem. Soc. 139, 2794 (2017).

${ }^{15}$ S. Mishra, V. S. Poonia, C. Fontanesi, R. Naaman, A. M. Fleming, and C. J. Burrows, J. Am. Chem. Soc. 141, 123 (2019).

${ }^{16}$ S. Mishra, A. K. Mondal, S. Pal, T. K. Das, E. Z. B. Smolinsky, G. Siligardi, and R. Naaman, J. Phys. Chem. C 124, 10776 (2020).

${ }^{17}$ S. Mishra, S. Pirbadian, A. K. Mondal, M. Y. El-Naggar, and R. Naaman, J. Am. Chem. Soc. 141, 19198 (2019).

${ }^{18}$ S. Ghosh, S. Mishra, E. Avigad, B. P. Bloom, L. T. Baczewski, S. Yochelis, Y. Paltiel, R. Naaman, and D. H. Waldeck, J. Phys. Chem. Lett. 11, 1550 (2020).

${ }^{19}$ T. S. Metzger, S. Mishra, B. P. Bloom, N. Goren, A. Neubauer, G. Shmul, J. Wei, S. Yochelis, F. Tassinari, C. Fontanesi, D. H. Waldeck, Y. Paltiel, and R. Naaman, Angew. Chem. Int. Ed. 59, 1653 (2020).

${ }^{20}$ M. Gazzotti, S. Arnaboldi, S. Grecchi, R. Giovanardi, M. Cannio, L. Pasquali, A. Giacomino, O. Abollino, and C. Fontanesi, Electrochim. Acta 286, 271 (2018).

${ }^{21}$ P. C. Mondal, W. Mtangi, and C. Fontanesi, Small Methods 2, 1700313 (2018).

${ }^{22}$ J. A. Switzer, H. M. Kothari, P. Poizot, S. Nakanishi, and E. W. Bohannan, Nature 425, 490 (2003).

${ }^{23}$ D. Avnir, Adv. Mater. 30, 1706804 (2018).

${ }^{24}$ L. Durán Pachón, I. Yosef, T. Z. Markus, R. Naaman, D. Avnir, and G. Rothenberg, Nat. Chem. 1, 160 (2009).

${ }^{25}$ See https://en.wikipedia.org/w/index.php?title=Nickel_electroplating\&oldid=9 92483061 for "Nickel electroplating" (2020).

${ }^{26}$ A. Hoek and W. M. H. Sachtler, J. Catal. 58, 276 (1979). 